\title{
Kinematic distances of pre-main-sequence stars in the Lupus star-forming region
}

\author{
P. A. B. Galli, ${ }^{1}$ R. Teixeira,${ }^{1}$ C. Ducourant,${ }^{2}$ and C. Bertout ${ }^{3}$ \\ ${ }^{1}$ Instituto de Astronomia, Geofísica e Ciências Atmosféricas, Universidade de São Paulo, \\ Rua do Matão, 1226 Cidade Universitária, 05508-900 São Paulo SP, Brazil \\ email: galli@astro.iag.usp.br \\ ${ }^{2}$ Observatoire Aquitain des Sciences de l'Univers, CNRS-UMR 5804, BP 89, 33270 Floirac, \\ France \\ ${ }^{3}$ Institut d'Astrophysique de Paris, 98bis, Bd. Arago, 75014 Paris, France
}

\begin{abstract}
To reliably determine the main physical parameters (masses and ages) of young stars, we must know their distances. While the average distance to nearby star-forming regions $(<300 \mathrm{pc})$ is often known, the distances to individual stars are usually unknown. Individual distances to members of young moving groups can be derived from their radial velocities and proper motions using the convergent-point strategy. We investigate the kinematic properties of the Lupus moving group with the primary objective of deriving individual distances to all group members.
\end{abstract}

Keywords. stars: distances, stars: kinematics, stars: pre-main-sequence, ISM: individual (Lupus star-forming region)

\section{Introduction}

The Lupus dark cloud complex is a low-mass star-forming region that contains one of the richest associations of $\mathrm{T}$ Tauri stars. While the precise distance to the Lupus complex is still being debated, little recent progress has been made as regards distance determinations to individual stars in this region, such as the faint pre-main-sequence (PMS) stars. Although average distances to star-forming regions provide valuable information, what we really need to determine the main physical parameters (masses and ages) of stars are the distances to individual stars. The vast majority of PMS stars in Lupus were not observed by the Hipparcos satellite because of their faint magnitudes, nor do they have any trigonometric parallaxes measured from the ground owing to their distances. However, an alternative method based on determination of the convergent point $(\mathrm{CP})$ of a co-moving group of stars and individual radial velocities exists and allows for the determination of individual parallaxes. This is what we are attempting to do here.

\section{A Sample of Pre-Main Sequence Stars in Lupus}

Our list of candidate members of the Lupus moving group consists of 258 PMS stars identified in previous surveys of this star-forming region (Krautter et al. 1997; Wichmann et al. 1997a,b; Coméron 2008; López-Martí et al. 2011). We obtained proper-motion data for these stars from the SPM4 (Girard et al. 2011), Tycho-2 (Høg et al. 2000) and Ducourant et al. (2005) catalogues. Fig. 1 shows the proper-motion vectors of Lupus stars and, as can be seen, they point towards a common direction. To develop our strategy, we took advantage of published radial velocities from various sources, which we 


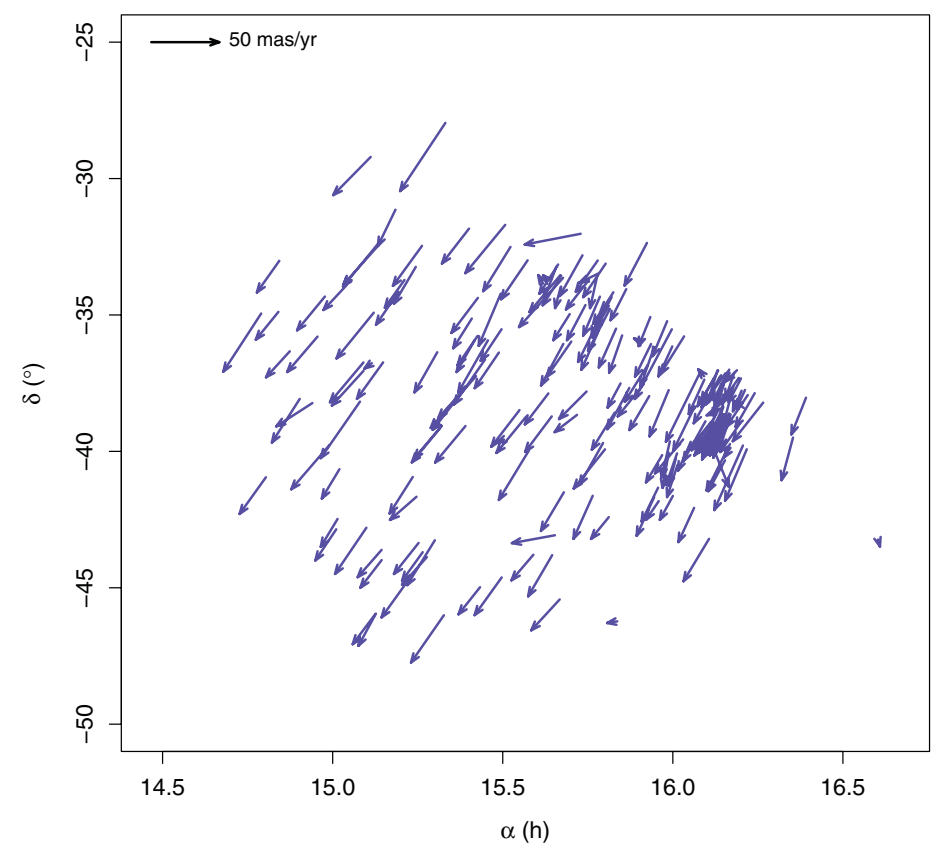

Figure 1. Proper-motion vectors of the Lupus candidate PMS stars.

supplemented with new measurements based on spectroscopic observations with FEROS on the ESO $2.2 \mathrm{~m}$ telescope (La Silla Observatory, Chile).

\section{Convergent-Point Analysis and Kinematic Parallaxes}

The technique we use to derive the CP position of the Lupus moving group is the new CP method (Galli et al. 2012), which was recently published. This strategy uses a maximum-likelihood analysis to simultaneously determine the CP position and the membership probabilities of the stars in the moving group.

When we apply the CP method to the sample of Lupus candidate members, we identify a moving group consisting of 114 stars. Among the 114 members, only 26 stars have reliable radial-velocity measurements, which we use to derive the individual parallaxes. These are given by

$$
\pi_{\text {ind }}=\frac{4.74 \mu_{\|}}{v_{\mathrm{r}} \tan \lambda},
$$

where $\mu_{\|}$is the stellar proper motion in the direction of the CP (see Galli et al. 2012), $v_{\mathrm{r}}$ is the radial velocity and $\lambda$ is the angular distance between the star and the CP.

For the remaining 88 stars with unknown radial velocities, we adopt the hypothesis that all group members share the same space motion to derive tentative parallaxes. We first determine the average spatial velocity, $v_{\text {space }}$, of the moving group based on the space velocity of the 26 stars with known radial velocities and then compute the approximate parallax as

$$
\pi_{\text {app }}=\frac{4.74 \mu_{\|}}{v_{\text {space }} \sin \lambda} .
$$

We compare the individual parallaxes using Eq. (3.1) and the approximate parallaxes 

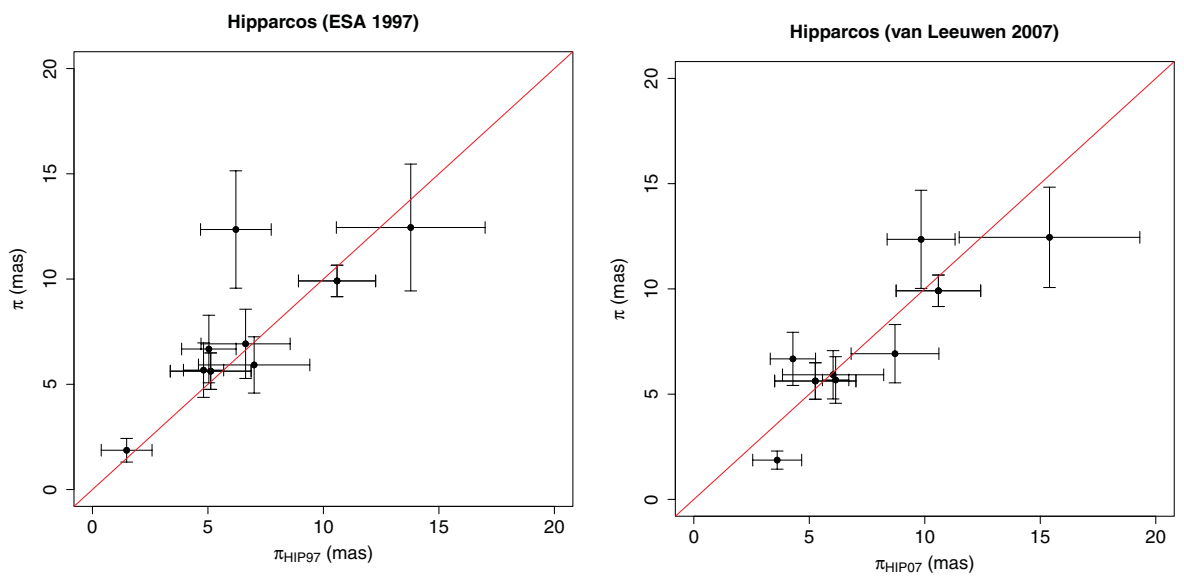

Figure 2. Parallaxes derived here compared with Hipparcos parallaxes.

based on Eq. (3.2) for the sample of 26 members with known radial velocities and conclude that both procedures return similar results (within the error bars).

\section{Comparison with Hipparcos results}

In Fig. 2 we compare the parallaxes derived here with the trigonometric parallaxes from the Hipparcos catalogue. We consider both versions of this catalogue: the original catalogue (ESA 1997; hereafter HIP97) and the new reduction of Hipparcos data (van Leeuwen 2007; hereafter HIP07). We found only nine Hipparcos stars among the 114 Lupus members selected in our CP analysis. The rms with respect to HIP97 and HIP07 is 2.28 and 1.92 mas, respectively. The mean differences between the parallaxes derived

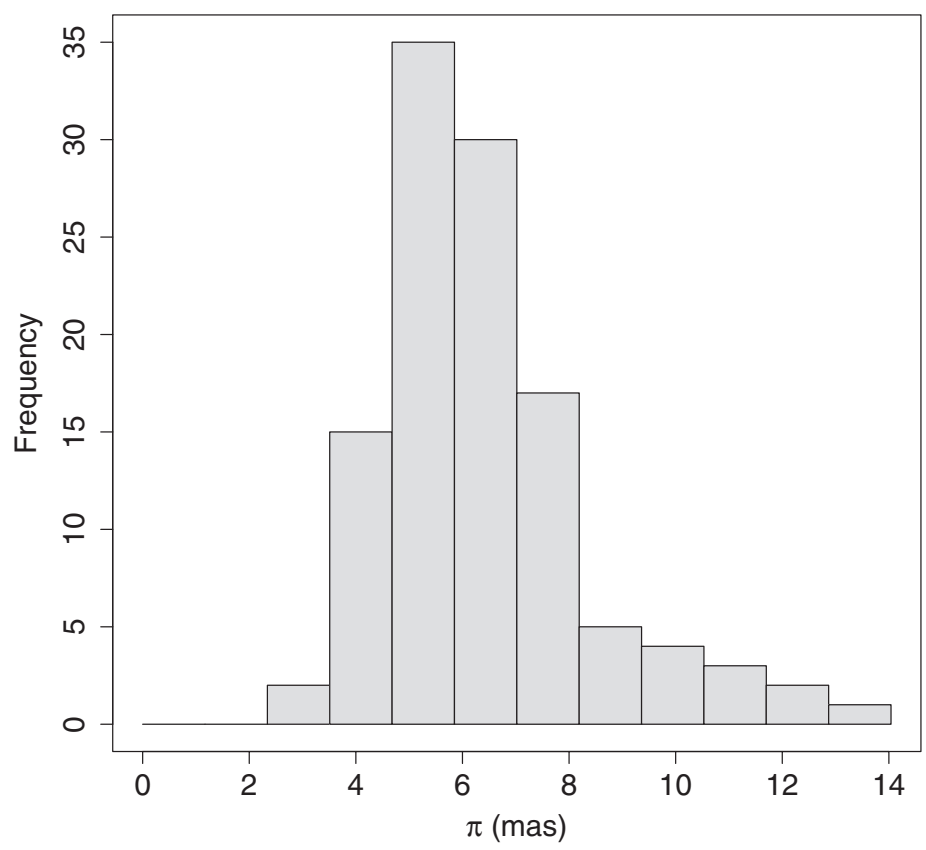

Figure 3. Parallax histogram of the 114 members of the Lupus moving group. 
here and those in HIP97 and HIP07 are -0.68 and 0.35 mas, respectively. We conclude that our results are in good agreement with the trigonometric parallaxes obtained by Hipparcos.

\section{Results}

Fig. 3 shows the histogram of parallaxes for the 114 members of the Lupus moving group. The average parallax and standard deviation are $\pi=6.4 \pm 1.9$ mas and the average error in the parallaxes is $\sigma_{\pi}=1.3$ mas. We note the presence of background and foreground stars, thus confirming that the Lupus complex occupies a large volume in space. The distances derived here will be used to investigate the physical properties (luminosities, masses and ages) of the Lupus stars.

\section{References}

Comerón, F. 2008, Handbook of Star Forming Regions, Vol. II, 295

Ducourant, C., Teixeira, R., Périé, J. P., et al. 2005, A\&A, 438, 769

ESA 1997, VizieR Online Data Catalog, 1239

Galli, P. A. B., Teixeira, R., Ducourant, C., Bertout, C., \& Benevides-Soares, P. 2012, A\&A, $538, \mathrm{~A} 23$

Girard, T. M., van Altena, W. F., Zacharias, N., et al. 2011, AJ, 142, 15

Høg, E., Fabricius, C., Makarov, V. V., et al. 2000, VizieR Online Data Catalog, 1259

Krautter, J., Wichmann, R., Schmitt, J. H. M. M., et al. 1997, A\&AS, 123, 329

López Martí, B., Jiménez-Esteban, F., \& Solano, E. 2011, AछA, 529, A108

van Leeuwen, F. 2007, $A \mathscr{\mho} A, 474,653$

Wichmann, R., Krautter, J., Covino, E., et al. 1997a, A\&\&A, 320, 185

Wichmann, R., Sterzik, M., Krautter, J., Metanomski, A., \& Voges, W. 1997b, A\&̋A, 326, 211 